nal therapeutic strategies can be developed for patients with recurrent early pregnancy loss. Therapies directed at innate inflammatory mediators, such as complement and TNF- $\alpha$, merit more intense scrutiny in pregnant women.

\section{Acknowledgments}

Work in the author's laboratory is supported by grants from the NIH (AR49772; AI55007), the Mary Kirkland Center for Lupus Research, and the Alliance for Lupus Research. Guillermina Girardi's critical review of the manuscript and invaluable help with the figures are gratefully acknowledged.

Address correspondence to: Jane E. Salmon, Hospital for Special Surgery, 535 East 70th Street, New York, New York 10021, USA. Phone: (212) 606-1422; Fax: (212) 717-1192; E-mail: salmonj@hss.edu.

1. Mellor, A.L., and Munn, D.H. 2000. Immunology at the maternal-fetal interface: lessons for $T$ cell tolerance and suppression. Annu. Rev. Immunol. 18:367-391.

2. Xu, C., et al. 2000. A critical role for murine complement regulator crry in fetomaternal tolerance.
Science. 287:498-501.

3. Mellor, A.L., et al. 2001. Prevention of T cell-driven complement activation and inflammation by tryptophan catabolism during pregnancy. Nat. Immunol. 2:64-68.

4. Aluvihare, V.R., Kallikourdis, M., and Betz, A.G. 2004. Regulatory $\mathrm{T}$ cells mediate maternal tolerance to the fetus. Nat. Immunol. 5:266-271.

5. Erlebacher, A., Zhang, D., Parlow, A.F., and Glimcher, L.H. 2004. Ovarian insufficiency and early pregnancy loss induced by activation of the innate immune system. J. Clin. Invest. 114:39-48. doi:10.1172/JCI200420645.

6. Caucheteux, S.M., Kanellopoulos-Langevin, C., and Ojcius, D.M. 2003. At the innate frontiers between mother and fetus: linking abortion with complement activation. Immunity. 18:169-172.

7. Tedesco, F., et al. 1993. Susceptibility of human trophoblast to killing by human complement and the role of the complement regulatory proteins. J. Immunol. 151:1562-1570.

8. Holers, V.M., et al. 2002. Complement C3 activation is required for antiphospholipid antibodyinduced fetal loss. J. Exp. Med. 195:211-220.

9. Girardi, G., et al. 2003. Complement C5a receptors and neutrophils mediate fetal injury in the antiphospholipid syndrome. J. Clin. Invest. 112:1644-1654. doi:10.1172/JCI200318817.

10. Schwaeble, W.J., and Reid, K.B. 1999. Does properdin crosslink the cellular and the humoral immune response? Immunol. Today. 20:17-21.

11. Wang, Y., et al. 2000. A role for complement in antibody-mediated inflammation: C5-deficient DBA/ 1 mice are resistant to collagen-induced arthritis. J. Immunol. 164:4340-4347.
12. Zwaka, T.P., et al. 2002. Complement and dilated cardiomyopathy: a role of sublytic terminal complement complex-induced tumor necrosis factoralpha synthesis in cardiac myocytes. Am. J. Pathol. 161:449-457.

13. Riedemann, N.C., et al. 2003. Regulation by C5a of neutrophil activation during sepsis. Immunity. 19:193-202.

14. Yui, J., Hemmings, D., Garcia-Lloret, M., and Guilbert, L.J. 1996. Expression of the human p55 and p75 tumor necrosis factor receptors in primary villous trophoblasts and their role in cytotoxic signal transduction. Biol. Reprod. 55:400-409.

15. Arck, P.C., Troutt, A.B., and Clark, D.A. 1997. Soluble receptors neutralizing TNF-alpha and IL-1 block stress-triggered murine abortion. Am. J. Reprod. Immunol. 37:262-266.

16. Mantzoros, C.S., et al. 1997. Leptin concentrations in relation to body mass index and the tumor necrosis factor-alpha system in humans. J. Clin. Endocrinol. Metab. 82:3408-3413.

17. Niswender, G.D., Juengel, J.L., Silva, P.J., Rollyson, M.K., and McIntush, E.W. 2000. Mechanisms controlling the function and life span of the corpus luteum. Physiol. Rev. 80:1-29.

18. Bornstein, S.R., Rutkowski, H., and Vrezas, I. 2004. Cytokines and steroidogenesis. Mol. Cell. Endocrinol. 215:135-141.

19. Raghupathy, R., et al. 2000. Cytokine production by maternal lymphocytes during normal human pregnancy and in unexplained recurrent spontaneous abortion. Hum. Reprod. 15:713-718.

20. Babbage, S.J., et al. 2001. Cytokine promoter gene polymorphisms and idiopathic recurrent pregnancy loss. J. Reprod. Immunol. 51:21-27.

\title{
Lonely in Paris: when one gene copy isn't enough
}

\section{Ramesh A. Shivdasani}

\begin{abstract}
Departments of Medical Oncology and Cancer Biology, Dana-Farber Cancer Institute; and Department of Medicine, Harvard Medical School,
\end{abstract} Boston, Massachusetts, USA.

\begin{abstract}
Circulating platelets are continually replenished by fragmentation of terminally differentiated megakaryocytes. Processes disrupted in inherited thrombocytopenias frequently shed light on normal thrombopoietic mechanisms. An especially rare condition called Paris-Trousseau syndrome (PTS) seems to occur by virtue of hemizygous loss of the FLI1 transcription factor gene. Provocative new data suggest that FLI1 shows monoallelic expression during a brief window in megakaryocyte differentiation, which thus explains the dominant inheritance pattern of PTS despite the presence of one normal FLI1 allele (see the related article beginning on page 77).
\end{abstract}

The rare familial thrombocytopenic disorder Paris-Trousseau syndrome (PTS), reported in fewer than a dozen patients, is associated with inherited deletion of a portion of the long arm of chromosome 11 (1). Blood platelets in affected individ-

Nonstandard abbreviations used: GATA binding protein 1 (GATA-1); Paris-Trousseau syndrome (PTS).

Conflict of interest: The author has declared that no conflict of interest exists.

Citation for this article: J. Clin. Invest. 114:17-19 (2004) doi:10.1172/JCI200422292. uals have a normal lifespan in the circulation; however, the number of bone marrow megakaryocytes is greatly increased, whereas megakaryocyte maturation is arrested, and many cells apparently fail to survive into terminal stages of differentiation $(1,2)$. Together these findings suggest that platelet release is substantially impaired. Megakaryocyte release of newly synthesized platelets, a process unique to mammals, occurs through a dramatic morphogenetic transition $(3,4)$. Nearly the entire cytoplasm converts into lengthy beaded projections (proplatelets), within which organelles are transported along unique microtubule tracks and nascent blood platelets assemble de novo (5) (Figure 1). The use of thrombopoietin, a megakaryocyte growth factor, to expand cells ex vivo and incisive mouse models of thrombocytopenia has led to rapid progress in understanding genetic and cellular mechanisms of platelet biogenesis (6); the emerging appreciation has important implications for diseases in which hemorrhage or thrombosis is a significant feature. In this issue of the JCI, Raslova and colleagues highlight transient monoallelic expression of a transcription factor gene en route to platelet release and explain why patients with PTS, who retain one normal copy of chromosome 11, produce few and defective platelets (7). Related gene regulatory mechanisms might well account for other dominantly inherited disorders in which haploinsufficiency or 


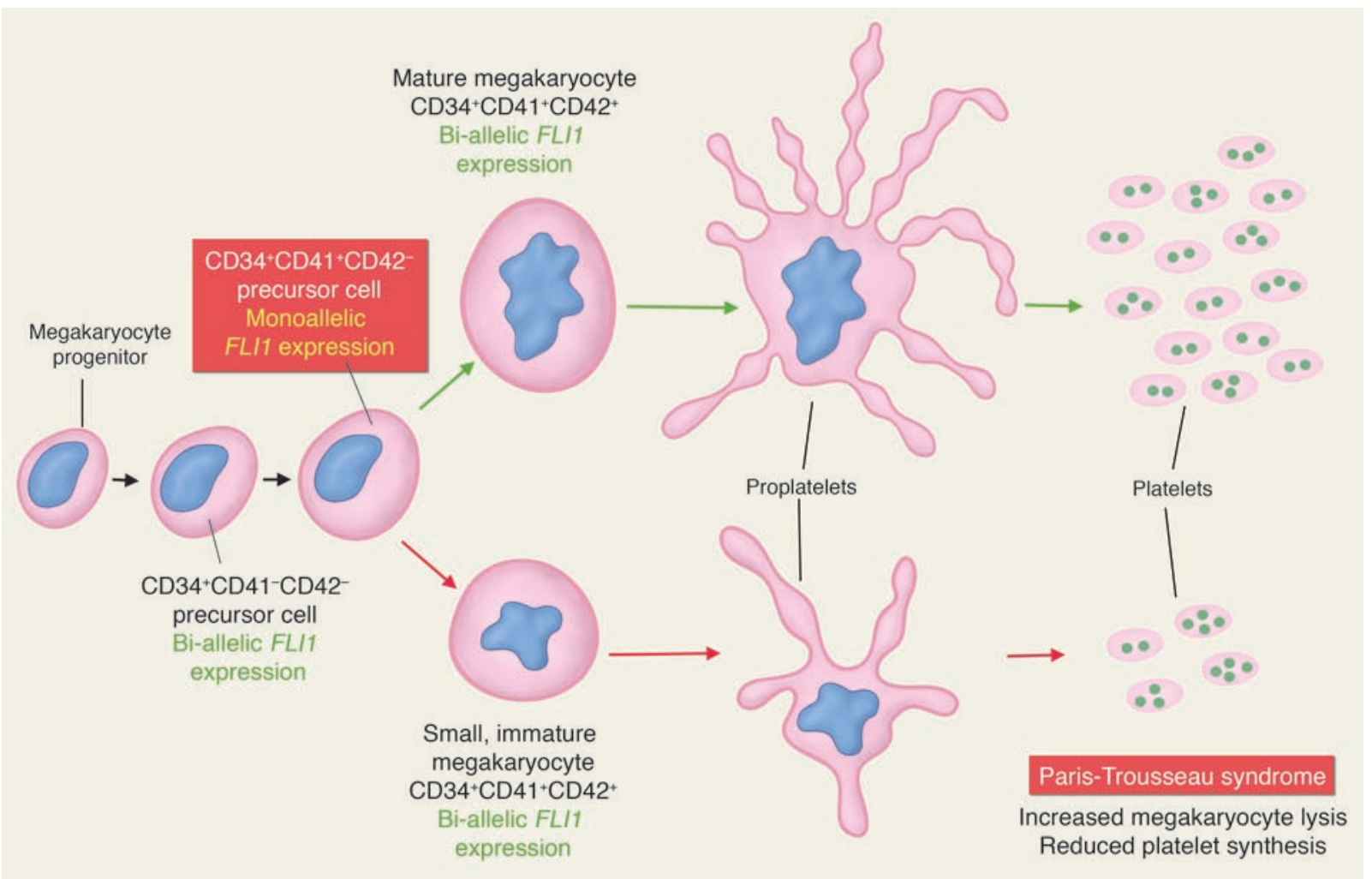

\section{Figure 1}

Schema of steps in megakaryocyte differentiation leading to platelet release in normal individuals and patients with PTS. Immature megakaryocyte progenitors, depicted on the left, acquire defining attributes (e.g., polyploidy) and surface markers (e.g., CD41) en route to a terminal step in which the cytoplasmic mass is converted into proplatelets and nascent blood platelets are assembled and released. Megakaryocytes expressing CD41 can be separated from more mature cells that also express the CD42 marker. Raslova et al. (7) propose that the transition between these two stages is marked by transient monoallelic expression of the $F L / 1$ gene, an event that is presumed to result in normal cell differentiation, as illustrated in the top right branch of this figure. In PTS, where patients carry a multigene 11q deletion, absence of one FLI1 allele produces a population of megakaryocytes that is transiently devoid of any FL/1 transcription. The authors infer that it is this deficit that results in subpopulations of micromegakaryocytes and morphologically abnormal platelets as well as thrombocytopenia, as shown in the bottom right branch. The model potentially explains the genetic and major clinicopathologic features of PTS.

interference with the wild-type product are inadequate explanations.

\section{A transcriptional basis for PTS}

Undeterred by the extreme rarity of PTS, Raslova et al. (7) have approached important questions raised by this unusual disorder. First, of the genes encoded within the 11q23-q24 deletion interval, the authors focused on the atypical ETS-family transcription factor FLI1 (8). Many megakaryocytic gene promoters harbor closely spaced GATA- and ETS-binding sites, suggesting a role for some ETS protein(s) in lineagespecific gene expression (9). Although FLI1 is one of several such factors, genetic studies previously had provided a vital clue in the phenotype of Fli1-knockout mice: increased numbers of megakaryocyte progenitors, with morphologic defects that, to a first approximation, resemble those seen in PTS patients; and reduced expression of several megakaryocytic genes (10, 11). FLI-1 also interacts physically with GATA binding protein 1 (GATA-1), and in vitro, the two transcription factors activate megakaryocytic genes synergistically (12). FLI1 is hence an especially attractive candidate for a key transcriptional regulator of megakaryocyte differentiation, and Raslova et al. demonstrate that FLI1 overexpression rescues critical defects in cells derived from PTS patients.

\section{A novel explanation for dominant inheritance of PTS}

If FLI1 deficiency is the central lesion in PTS megakaryocytes, as this result implies, why does the wild-type FLI1 allele fail to compensate for hemizygous gene loss? While the importance of gene dosage represents one obvious explanation, Raslova et al. were influenced by the curious observation that PTS patients carry two discrete populations of megakaryocytes and platelets: many abnormally small megakaryocytes (micromegakaryocytes) and platelets (up to $15 \%$ of the total) that harbor giant $\alpha$-granules and respond poorly to thrombin $(1,2)$. Reasoning that these morphologically distinct populations might derive from precursors in which FLI1 is expressed differentially at some point in the cellular ontogeny, they applied RNA-FISH and single-cell RT-PCR to demonstrate transient monoallelic FLI1 expression during a narrow window in megakaryocyte differentiation (Figure 1). The results imply that hemizygous FLI1 loss in patients with PTS would leave some fraction of maturing megakaryocytes with too little FLI1, albeit transiently, a deficit that could result in the morphologic dichotomy and thrombocytopenia. 
Monoallelic expression of autosomal genes is associated classically with genomic imprinting during embryogenesis or with allelic exclusion of surface receptor and selected cytokine genes in lymphocytes and olfactory neurons (13-16). However, rigorous demonstration of monoallelic gene expression requires methods that are not standard in most laboratories, so the extent of the phenomenon conceivably is underestimated. The $11 \mathrm{q}$ deletion in PTS can originate in either parent, and other disease features establish that FLI1 is not an imprinted locus. The observations of Raslova et al. (7) thus represent an apparently novel mechanism for transient regulation of a transcription factor gene. Before considering its significance and mechanisms, however, it is helpful to recall that previous demonstration of the same phenomenon for the Pax5 gene in B lymphocytes (17) is controversial (18), largely because of the challenges in executing and interpreting single-cell RT-PCR reactions. It is therefore important to verify the FLI1 claim, ideally by examining protein levels at discrete stages in megakaryocyte maturation: depending on protein turnover rates, one prediction is that in individual wild-type CD41+CD42- megakaryocytes, FLI1 expression is halved, whereas some PTS-derived cells express no FLI1 at the same juncture. Another hypothesis that follows logically is that $\mathrm{CD} 41^{+}$megakaryocytes manifest an FLI1-dependent differentiation "checkpoint" somewhere along the transition that precedes CD42 expression (Figure 1).

If the Raslova model withstands further scrutiny, it raises very interesting questions. The mechanisms underlying transient monoallelic gene expression in this example are unknown and likely consist of some combination of the numerous epigenetic modifications that repress transcription. It is perhaps more interesting to ponder the possible reasons for a special- ized cell to enable monoallelic gene expression during a brief period in its complex life cycle. Normally such regulation would merely adjust transcript levels; only in rare instances such as PTS would it produce unintended, deleterious consequences. One possibility is that FLI1 allelic exclusion reflects an adaptation of stochastic or pulsatile transcriptional activation, putative general features of gene regulation $(19,20)$, which may transiently be skewed to physiologic advantage at selected loci. Regardless of the rationale and molecular mechanisms, monoallelic gene expression in mature megakaryocytes, which have a significantly polyploid DNA content, demands special consideration. Thus, the Raslova model implies that each of the 16 or more chromosome copies derived from one of the two original sets is epigenetically and selectively modified for transient silencing of some genes. Addressing these and related questions should stimulate research in both platelet biogenesis and gene regulatory mechanisms. The Raslova study thus illustrates how astute and dogged investigation of rare clinical disorders still carries the potential to reveal underlying principles in both normal and aberrant cellular physiology.

Address correspondence to: Ramesh A. Shivdasani, Dana-Farber Cancer Institute, 44 Binney Street, Boston, Massachusetts 02115, USA. Phone: (617) 632-5746; Fax: (617) 632-4471; E-mail: ramesh_shivdasani@dfci.harvard.edu.

1. Breton-Gorius, J., et al. 1995. A new congenital dysmegakaryopoietic thrombocytopenia (Paris-Trousseau) associated with giant platelet alpha-granules and chromosome 11 deletion at $11 \mathrm{q} 23$. Blood. 85:1805-1814

2. Favier, R., et al. 2003. Paris-Trousseau syndrome: clinical, hematological, molecular data of ten new cases. Thromb. Haemost. 90:893-897.

3. Radley, J.M., and Scurfield, G. 1980. The mechanism of platelet release. Blood. 56:996-999.

4. Choi, E.S., Nichol, J.L., Hokom, M.M., Hornkohl, A.C., and Hunt, P. 1995. Platelets generated in vitro from proplatelet-displaying human megakaryocytes are functional. Blood. 85:402-413.

5. Italiano, J.E., Jr., Lecine, P., Shivdasani, R.A., and Hartwig, J.H. 1999. Blood platelets are assembled principally at the ends of proplatelet processes produced by differentiated megakaryocytes. J. Cell. Biol. 147:1299-1312.

6. Italiano, J.E., Jr., and Shivdasani, R.A. 2003. Megakaryocytes and beyond: the birth of platelets. J. Thromb. Haemost. 1:1174-1182.

7. Raslova, H., et al. 2004. FLI1 monoallelic expression combined with its hemizygous loss underlies ParisTrousseau/Jacobsen thrombopenia. J. Clin. Invest. 114:77-84. doi:10.1172/JCI200421197.

8. Zhang, L., et al. 1993. The Fli-1 proto-oncogene, involved in erythroleukemia and Ewing's sarcoma, encodes a transcriptional activator with DNAbinding specificities distinct from other Ets family members. Oncogene. 8:1621-1630.

9. Lemarchandel, V., Ghysdael, J., Mignotte, V., Rahuel, C., and Romeo, P.-H. 1993. GATA and Ets cis-acting sequences mediate megakaryocyte-specific expression. Mol. Cell. Biol. 13:668-676.

10. Hart, A., et al. 2000. Fli-1 is required for murine vascular and megakaryocytic development and is hemizygously deleted in patients with thrombocytopenia. Immunity. 13:167-177.

11. Kawada, H., et al. 2001. Defective megakaryopoiesis and abnormal erythroid development in Fli-1 genetargeted mice. Int. J. Hematol. 73:463-468.

12. Eisbacher, M., et al. 2003. Protein-protein interaction between Fli-1 and GATA-1 mediates synergistic expression of megakaryocyte-specific genes through cooperative DNA binding. Mol. Cell. Biol. 23:3427-3441.

13. Pernis, B., Chiappino, G., Kelus, A.S., and Gell, P.G. 1965. Cellular localization of immunoglobulins with different allotypic specificities in rabbit lymphoid tissues. J. Exp. Med. 122:853-876.

14. Nicholls, R.D., Knoll, J.H., Butler, M.G., Karam, S., and Lalande, M. 1989. Genetic imprinting suggested by maternal heterodisomy in nondeletion Prader-Willi syndrome. Nature. 342:281-285.

15. Chess, A., Simon, I., Cedar, H., and Axel, R. 1994. Allelic inactivation regulates olfactory receptor gene expression. Cell. 78:823-834.

16. Bix, M., and Locksley, R.M. 1998. Independent and epigenetic regulation of the interleukin- 4 alleles in CD4+ T cells. Science. 281:1352-1354.

17. Nutt, S.L., et al. 1999. Independent regulation of the two Pax5 alleles during B-cell development. Nat. Genet. 21:390-395.

18. Rhoades, K.L., et al. 2000. Allele-specific expression patterns of interleukin- 2 and Pax- 5 revealed by a sensitive single-cell RT-PCR analysis. Curr. Biol. 10:789-792.

19. Newlands, S., et al. 1998. Transcription occurs in pulses in muscle fibers. Genes Dev. 12:2748-2758.

20. Fiering, S., Whitelaw, E., and Martin, D.I. 2000. To be or not to be active: the stochastic nature of enhancer action. Bioessays. 22:381-387. 